\title{
Hip fracture care and mortality among patients treated in dedicated COVID-19 and non-COVID-19 circuits
}

\author{
Cristina Ojeda-Thies ${ }^{1}\left[\right.$ - Javier Cuarental-García ${ }^{1}$ - Elena García-Gómez ${ }^{2}$ - Carlos Hugo Salazar-Zamorano ${ }^{3}$. \\ Javier Alberti-Maroño ${ }^{3}$. Luis Rafael Ramos-Pascua ${ }^{1,4}$
}

Received: 6 December 2020 / Accepted: 19 January 2021 / Published online: 7 February 2021

(c) European Geriatric Medicine Society 2021

\section{Key summary points}

Aim What were the effects of the COVID-19 pandemic and patient cohorting on the mortality and care provided for patients with hip fractures?

Findings Mortality due to COVID-19 infection was higher before patient cohorting, and no nosocomial infections were detected after cohorting. Survival of patients treated in the COVID-19 circuit was similar to non-COVID-19 controls, in spite of longer surgical delay and length of stay.

Message Separate circuits for COVID-19 and non-COVID-19 patients allows adequate hip fracture care, without observing increased mortality when delaying surgery until stabilization among patients with severe respiratory illness.

\begin{abstract}
Introduction To analyze the effect of the COVID-19 pandemic on the provision of fragility hip fracture care, comparing patients treated before cohorting and in separate COVID-19 and non-COVID-19 circuits with the corresponding months in 2018 and 2019.

Materials and methods Retrospective single-center cohort study including 64 patients with fragility hip fractures treated during the COVID-19 pandemic (March 1st-May 1st, 2020), compared to 172 patients treated in 2018 and 2019. Dedicated COVID-19 and non-COVID-19 circuits were established on March 14th. Patients treated before cohorting (17 patients), in COVID-19 (14 patients) and non-COVID-19 circuits (33 patients) were included.

Results Baseline characteristics were similar for 2018-19 and 2020. Patients in 2020 had a lower median surgical delay (50.5 vs. $91.3 \mathrm{~h}$ ) and length of stay (9.0 vs. 14.0 days), while those with COVID-19, had longer surgical delays and length of stay ( $87.7 \mathrm{~h}$ and 15.0 days, respectively). Thirty-days mortality was higher among patients before cohorting, but similar in Covid-19 and non-Covid-19 pathways compared to $2018-19$ (7.1\% and 3.0\% vs 5.2\%, respectively). $23.5 \%$ of patients treated before circuiting suffered coronavirus infectious disease-19 disease after discharge. Following separation, no secondary cases of coronavirus infectious disease-19 were observed.

Conclusions Separate circuits for patients with and without coronavirus infectious disease-19 provided adequate hip fracture care. We did not observe increased mortality rates among hip fracture patients with preoperatively confirmed or suspected coronavirus infectious disease-19, compared to negative cases and 2018-19. Delaying surgery among patients with severe respiratory illness until a favourable trend could be observed did not lead to increased mortality.
\end{abstract}

Keywords Hip fracture $\cdot$ Care pathways $\cdot$ COVID-19 $\cdot$ Mortality $\cdot$ Cohorting precautions

Cristina Ojeda-Thies

cristina.ojeda@salud.madrid.org

1 Department of Traumatology and Orthopedic Surgery, Hospital Universitario 12 de Octubre, Avda Córdoba s/n, 28041 Madrid, Spain

2 Geriatric Medicine, Hospital Universitario 12 de Octubre, Madrid, Spain
3 Anaesthesiology and Postoperative Care, Hospital Universitario 12 de Octubre, Madrid, Spain

4 Department of Surgery, School of Medicine, Universidad Complutense de Madrid, Madrid, Spain 


\section{Introduction}

The COVID-19 pandemic has profoundly disrupted the provision of orthopedic care worldwide. In regions with a high case volume, healthcare systems became overwhelmed, compromising the care of other diseases [1,2]. During lockdown, the volume of major trauma and activity-related injuries decreased more than hip fractures [3, 4]. COVID-19 mortality is higher among older patients and in those with comorbidities, particularly the type of patient likely to suffer a hip fracture, and it is essential to avoid COVID-19 cross-infection among patients admitted for hip fractures. Recent studies have described a sevenfold increase in mortality in patients with hip fractures concomitantly affected by COVID-19, with approximately one-third of patients dying by the first month [5]. Many of these studies are not adjusted for age, sex or comorbidities and do not take other factors into account such as surgical delay, or are limited to data collected during 2020 without comparison with results from previous years [1, 6-11].

Pandemics have been characterized by waves of activity spread over months, and we are currently in a second wave, as predicted by modeling studies [12]. It is important to establish circuits to provide safe and effective care for fragility hip fractures in spite of the coronavirus pandemic. Hip fracture care involves medical, surgical, rehabilitative and emergency care and is considered a good indicator for the overall performance of hospital services, with several international indicators, particularly surgical delay and mortality [13]. Provision of adequate hip fracture care in spite of the stress due to COVID-19 suffered by healthcare systems can be interpreted as an indicator of the quality of care provided for non-elective non-coronavirus infectious disease-19 during the pandemic, as it involves all the aforementioned elements of care. Measuring the effect of concomitant coronavirus infectious disease-19 on patients suffering fragility hip fractures is also of interest.

The goal of this study was to analyze the effect of the first wave of the COVID-19 pandemic on the provision of fragility hip fracture care comparing patients treated before establishing dedicated circuits and in separate COVID-19 and non-COVID-19 circuits, compared with the corresponding months during the previous two years.

\section{Materials and methods}

\section{Study design}

We performed a cohort study including all patients admitted for fragility proximal femoral fractures (OTA/AO fracture classification $31 \mathrm{~A}, 31 \mathrm{~B}$, and $32(\mathrm{~A}-\mathrm{C})$ ) in a single hospital between March 1st, 2020 and May 1st, 2020 (Fig. 1), when the first wave of the COVID-19 pandemic overwhelmed the local healthcare system, compared with the corresponding months of 2018 and 2019. The two preceding years were chosen to avoid spurious associations and increase statistical power. High-energy injuries, periprosthetic and pathologic fractures were excluded. We obtained institutional review board approval for this study, defined as non-intervention by the Spanish Medicines Agency (Agencia Española de Medicamentos y Productos Sanitarios, AEMPS).

\section{Healthcare provision in Spain during the COVID-19 pandemic}

The first reported death due to coronavirus infectious disease-19 in Madrid occurred on March 3rd, 2020, in a 99-year old female admitted from a nursing home, though phylogeographic analysis suggests the SARS-CoV-2 pathogen was
Fig. 1 Patient flowchart during the period analysed
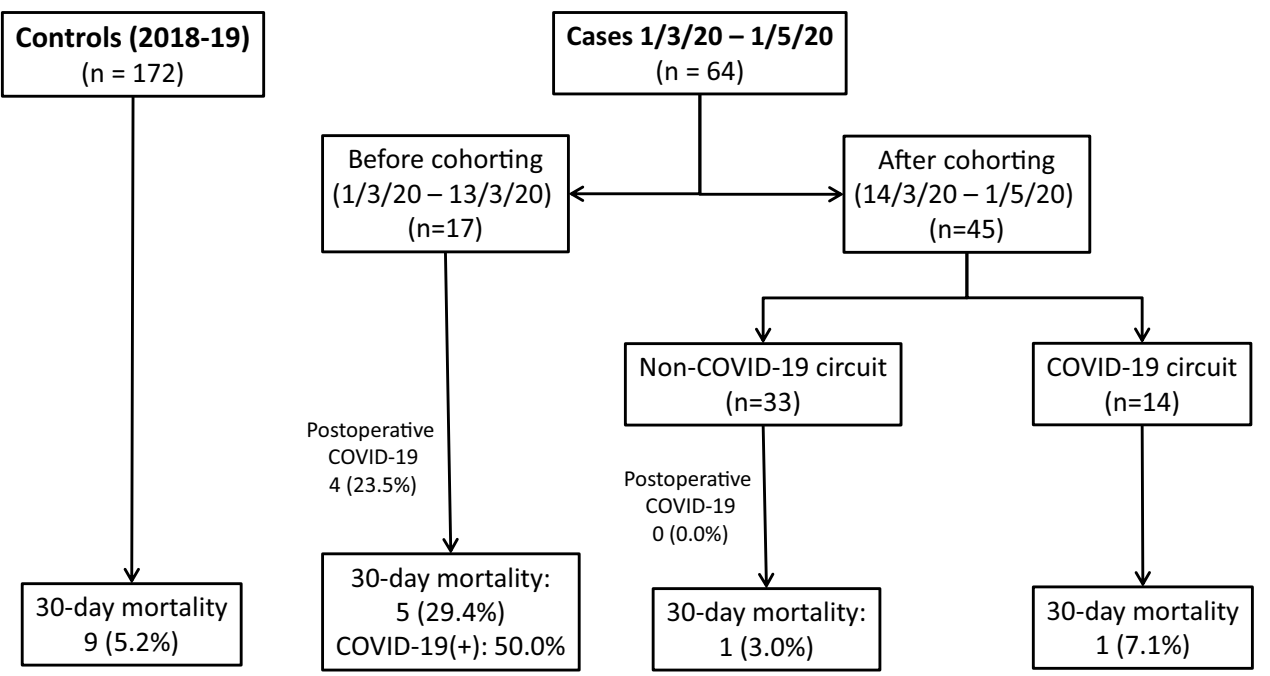
likely to have been circulating in Spain by mid-February 2020 [14]. Senior centers were isolated in the first week of March and visits to elderly care facilities halted. A nationwide State of Alarm was proclaimed on March 14th, 2020 with the cessation of all non-essential activities. Leaving the house was only permitted for essential workers or for purchasing basic items, and those 70 years or older were not allowed to abandon their homes for any leisure activity until May 2nd, 2020.

Healthcare is provided in Spain with a public healthcare system financed indirectly through taxation and managed by the autonomous regional governments. Each region is subdivided into basic heath zones including approximately 20.000 inhabitants that share a designated hospital for advanced care; thus, hospitals have defined catchment areas and populations [15]. Occupancy by COVID-19 cases alone reached $100 \%$ of pre-existing acute care beds by March 28th, and additional beds were placed in improvised areas such as congress venues, physical therapy gyms, outpatient clinics, and tents outside hospitals [16]. ICU bed occupancy reached nearly $300 \%$ and anaesthetic care units, paediatric and cardiac ICUs were repurposed for COVID-19 adult patients; makeshift ICUs were created in operating rooms and day surgery units. The COVID-19 caseload interrupted almost all non-COVID surgical and medical hospital activities for all hospitals in Madrid. The university hospital in which this study was performed is an urban Level 1 Trauma Centre with nearly 1300 beds and a catchment area of almost 450.000 people of which approximately 75.000 are aged 65 years and above.

\section{Case definitions and variables}

Baseline demographic, injury and surgical data (sex, age, place of residence, fracture type, type of anaesthesia and surgery) were collected for all patients, as well as pre-fracture functional independence for activities of daily living [Independent (Katz 5-6 points), partially dependent (Katz 3-4 points), severely dependent (Katz $0-2$ points) [17]], cognitive status (defining cognitive impairment as a score of more than 3 errors in Pfeiffer's Short Portable Mental Status Questionnaire [18]), and anaesthetic risk using the American Society of Anaesthesiologists (ASA) score as an indirect measure of comorbidity [19]. The surgical delay was measured as the time in hours from presentation in the emergency department to initiation of anaesthetic measures, and the length of stay as days between the day of admission and the day of discharge. Survival was determined by linkage of electronic hospital records and primary healthcare records, as well as by follow-up contact by telephone or in the clinic.

COVID-19 status was defined by microbiological diagnosis (positive detection of SARS-CoV-2 genome using polymerase chain reaction tests (RT-PCR) of nasopharyngeal swabs) and/or clinically suggestive symptoms and radiologic features. Patients with clinically suggestive symptoms and radiographic findings were considered to have pneumonia due to SARS-CoV-2 in spite of negative tests given the epidemiologic context of COVID-19 infection in Madrid, Spain, during those months with the estimated SARS-CoV-2 seroprevalence among the population of Madrid over $10 \%$ by the end of our 2-month inclusion period [20]. Nasopharyngeal swabs were taken for all patients admitted to hospital since March 14th, 2020, immediately prior to surgery for all patients already admitted, as well as for all discharged to nursing homes or geriatric rehabilitation units.

\section{Statistical analysis}

Continuous variables are reported using means and standard deviations if they show a normal distribution using histograms, P-P plots and the Kolmogorov-Smirnov test, and median and interquartile ranges if they seem non-normal. Continuous variables were compared using Student's $t$-test for independent samples and ANOVA or Mann-Whitney's $U$ and Kruskal-Wallis tests for independent samples, and $\chi^{2}$ and Fisher's exact tests for categorical variables. Significance was set at $p<0.05$. Data were analysed using SPSS version 20.0 (IBM, Armonk, New York, USA).

\section{Treatment circuits during the COVID-19 pandemic}

Patients were admitted into separate wards and operation theatres depending on clinical and microbiological COVID19 status from March 14th onwards. Patients in both groups were cohorted into double or triple rooms depending on availability. Those in the non-COVID19 circuit received co-managed orthogeriatric care. Patients in the COVID19 circuit were treated by clinicians from several medical specialties for their coronavirus infection, and by a single consultant orthopaedic surgeon who coordinated ward care, surgical programming, physiotherapy and discharge.

All patients in the COVID-19 circuit were operated on in a designated surgical theatre, with two consultant anaesthesiologists performing all anaesthetic procedures in this circuit. Patients were declared fit for surgery if they had less than $37.8^{\circ} \mathrm{C}$ fever and had no frank respiratory insufficiency, or required only low-dose oxygen through the nasal cannula. All patients received neuraxial anaesthesia with a weight-adjusted dose $1-1.5 \mathrm{ml}$ of intraspinal $0.5 \%$ plain bupivacaine at L3-L4 level. Patients with intracapsular fractures received cemented bipolar hemiarthroplasties or internal fixation. Patients with extracapsular fractures were treated with cephalomedullary nails. A consultant attending surgeon supervised or performed all surgeries. These circuits followed the guidelines of national and international societies for Anaesthesia [21], Surgery [22], Geriatrics and 
Osteoporotic Fractures [23]. Patients with COVID-19 pneumonia not fit for surgery received iliofascial blocks with $15 \mathrm{ml}$ of $0.125 \%$ Levobupivacaine and $4 \mathrm{mg}$ Dexamethasone using anatomic landmarks repeated as necessary and were mobilized out of bed as much as tolerated to improve respiratory function. Operated patients were mobilized out of bed the day of or after surgery, and commenced physiotherapy on the first postoperative day, with weight-bearing as tolerated. All patients received thromboprophylaxis with daily low-molecular weight heparin (LMWH) at prophylactic doses unless there was an indication of therapeutic anticoagulation.

\section{Results}

Table 1 summarizes the results observed for cases treated in 2020 compared to 2018-2019. Baseline demographic characteristics, residential, functional and cognitive status were similar for both observation periods, as was fracture type, anaesthetic management, and postoperative mobilisation. During the COVID-19 pandemic, surgical delay for hip fractures was significantly lower, as was also length of stay; destination at discharge was mainly back to private homes, at the expense of geriatric rehabilitation units; though 30- and 90-day mortality was higher in 2020 compared to 2018-2019, the difference did not reach statistical significance.
Table 1 Comparison of the casemix, management, and outcomes of patients treated for hip fracture in 2018-19 versus 2020

\begin{tabular}{|c|c|c|c|}
\hline & $\begin{array}{l}2018-2019 \\
(n=172)\end{array}$ & $\begin{array}{l}2020 \\
(n=64)\end{array}$ & $p$ value \\
\hline Age, years (SD) & $84.1(7.8)$ & $83.9(9.8)$ & 0.837 \\
\hline Sex, female $(\%)$ & $114(66.3)$ & $42(65.6)$ & 0.925 \\
\hline \multicolumn{4}{|l|}{ Residential status (\%) } \\
\hline Nursing care & 20 (11.6) & $7(10.9)$ & 0.307 \\
\hline \multicolumn{4}{|l|}{ Functional status (\%) } \\
\hline Independent & $98(57.0)$ & $32(50.0)$ & 0.479 \\
\hline Partially dependent & $46(26.8)$ & $23(35.9)$ & \\
\hline Dependent & $28(16.3)$ & $9(14.1)$ & \\
\hline \multicolumn{4}{|l|}{ Cognitive status (\%) } \\
\hline Cognitive impairment & $55(32.2)$ & $21(32.8)$ & 0.925 \\
\hline \multicolumn{4}{|l|}{ Anaesthetic risk score (\%) } \\
\hline ASA 3 or above & $128(74.4)$ & $57(89.1)$ & 0.015 \\
\hline Anticoagulation/antiaggregation (\%) & & & 0.706 \\
\hline Overall & $52(30.1)$ & $16(25.0)$ & \\
\hline Acenocumarol & $29(16.9)$ & $11(17.2)$ & \\
\hline DOACs & $9(5.2)$ & $2(3.1)$ & \\
\hline Clopidogrel/Ticagrelor & $14(8.1)$ & $3(4.7)$ & \\
\hline \multicolumn{4}{|l|}{ Fracture type $(\%)$} \\
\hline Intracapsular (OTA 31-B) & $82(47.7)$ & $29(45.4)$ & 0.145 \\
\hline Intertrochanteric (OTA 31-A) & $80(46.5)$ & $30(46.9)$ & \\
\hline Subtrochanteric (OTA 32(A-C)) & $10(5.8)$ & $5(7.8)$ & \\
\hline Spinal anaesthesia (\%) & $154(92.2)$ & $60(93.8)$ & 0.689 \\
\hline Mobilized by the first postoperative day (\%) & $36(20.9)$ & $13(20.3)$ & 0.917 \\
\hline \multicolumn{4}{|l|}{ Destination at discharge (\%) } \\
\hline Deceased in-hospital & $8(4.7)$ & $5(7.8)$ & 0.344 \\
\hline Geriatric rehabilitation unit & $81(47.1)$ & $4(6.2)$ & $<0.001$ \\
\hline Nursing home & $23(13.4)$ & $12(18.8)$ & 0.301 \\
\hline Private home & $60(34.9)$ & $43(67.2)$ & $<0,001$ \\
\hline Surgical delay, median hours (IQR) & $91.3(50.0-136.0)$ & $50.5(32.1-109.2)$ & 0.002 \\
\hline Length of stay, median days (IQR) & $14.0(10.0-19.0)$ & $9.0(6.3-15.5)$ & $<0.001$ \\
\hline \multicolumn{4}{|l|}{ Mortality (\%) } \\
\hline 30-day mortality & $9(5.2)$ & $7(10.9)$ & 0.121 \\
\hline 90-day mortality & $16(9.3)$ & $10(15.6)$ & 0.193 \\
\hline
\end{tabular}


The differences between the patients managed in the precohorting period (before organising circuits), the COVID-19 and the non-COVID-19 circuits during 2020 are shown in Table 2. Fourteen patients were treated in the COVID-19 circuit, nine with confirmed RT-PCR tests for COVID-19 preoperatively and four with symptoms and radiologic findings suggestive of COVID-19 pneumonia in spite of negative nasopharyngeal swabs. Another patient had atypical symptoms but an epidemiologic history and radiology suggestive of infection by SARS-CoV-2. Five of the nine RT-PCR $(+)$ cases had pneumonia, while two had only low-grade fever and two were completely asymptomatic. Four of the five patients considered clinically to have COVID-19 in spite of negative swabs had severe pneumonia, while one had typical radiologic features and only mild symptoms. Patients treated in the COVID-19 circuit tended to be older (mean age 86 years vs. 83 years) and slightly more likely to suffer cognitive impairment (36\% vs. $32 \%$ ) though without reaching statistical significance.

As can be expected among patients with concomitant pneumonia, surgical delay and length of stay were longer in COVID-19 than in non-COVID-19 patients ( $p=0.046$ and $p=0.008$, respectively). More patients in the COVID19 pathway were mobilised on the first postoperative day $(p=0.035)$. Mortality at 30 and 90 days was not significantly higher among patients treated in the COVID-19 pathway.

Four of the seventeen patients $(23.5 \%)$ treated before separating all patients into COVID-19 and non-COVID-19

Table 2 Comparison of the casemix, management and outcomes of patients treated for hip fracture in the 2020 pre-cohorting, non-COVID-19 and COVID-19 circuits

\begin{tabular}{|c|c|c|c|c|}
\hline & Pre-cohorting $(n=17)$ & $\begin{array}{l}\text { Non-COVID-19 } \\
\text { circuit }(n=33)\end{array}$ & COVID-19 circuit $(n=14)$ & $p$ value \\
\hline Age, years (SD) & $84.2(11.1)$ & $82.6(10.1)$ & $86.4(7.3)$ & 0.475 \\
\hline Sex, female $(\%)$ & $11(64.7)$ & $23(69.7 \%)$ & $8(57.1)$ & 0.706 \\
\hline \multicolumn{5}{|l|}{ Residential status (\%) } \\
\hline Nursing care & $2(11.8)$ & $3(9.1)$ & $4(28.6)$ & 0.203 \\
\hline \multicolumn{5}{|l|}{ Functional status (\%) } \\
\hline Independent & $9(52.9)$ & $16(48.6)$ & $7(50.0)$ & \multirow[t]{3}{*}{0.606} \\
\hline Partially dependent & $4(23.5)$ & $13(39.4)$ & $6(42.9)$ & \\
\hline Dependent & $4(23.5)$ & $4(12.1)$ & $1(7.1)$ & \\
\hline \multicolumn{5}{|l|}{ Cognitive status (\%) } \\
\hline Cognitive impairment & $6(35.3)$ & $10(30.3)$ & $5(35.7)$ & 0.907 \\
\hline \multicolumn{5}{|l|}{ Anaesthetic risk score (\%) } \\
\hline ASA 3 or above & $16(94.1)$ & $28(84.8)$ & $13(92.9)$ & 0.534 \\
\hline \multicolumn{5}{|l|}{ Anticoagulation/antiaggregation (\%) } \\
\hline Overall & $6(35.3)$ & $6(18.2)$ & $3(21.4)$ & \multirow[t]{4}{*}{0.178} \\
\hline Acenocumarol & $6(35.3)$ & $3(9.1)$ & $2(14.3)$ & \\
\hline DOACs & $0(0.0)$ & $2(6.1)$ & $0(0.0)$ & \\
\hline Clopidogrel/Ticagrelor & $0(0.0)$ & $2(6.1)$ & $1(7.1)$ & \\
\hline \multicolumn{5}{|l|}{ Fracture type $(\%)$} \\
\hline Intracapsular (OTA 31-B) & $7(41.2)$ & $15(45.4)$ & $7(50.0)$ & \multirow[t]{3}{*}{0.934} \\
\hline Intertrochanteric (OTA 31-A) & $9(52.9)$ & $15(45.5)$ & $6(44.9)$ & \\
\hline Subtrochanteric (OTA 32(A-C)) & $1(5.9)$ & $3(9.1)$ & $1(7.1)$ & \\
\hline Spinal anaesthesia (\%) & $16(100)$ & $32(97.0)$ & $12(100.0)$ & 0.537 \\
\hline Mobilized by the first postoperative day (\%) & $3(17.6)$ & $5(15.1)$ & $5(38.5)$ & $0.035^{*}$ \\
\hline \multicolumn{5}{|l|}{ Destination at discharge (\%) } \\
\hline Deceased in-hospital & $3(17.6)$ & $1(3.0)$ & $1(7.1)$ & 0.188 \\
\hline Geriatric rehabilitation unit & $1(5.9)$ & $2(6.1)$ & $1(7.1)$ & 0.987 \\
\hline Nursing home & $3(17.6)$ & $5(15.2)$ & $4(28.6)$ & 0.554 \\
\hline Private home & $10(58.8)$ & $25(75.8)$ & $8(57.1)$ & 0.319 \\
\hline Surgical delay, hours (IQR) & $48.4(23.3-73.3)$ & $51.1(40.9-98.0)$ & $87.7(46.2 .147 .2)$ & $0.046^{*}$ \\
\hline Length of stay, days (IQR) & $7.0(6.0-9.5)$ & $14.0(6.5-16.0)$ & $15.0(9.25-35.25)$ & $0.008^{*}$ \\
\hline Mortality (\%) & $5(29.4)$ & $1(3.0)$ & $1(7.1)$ & $0.016^{*}$ \\
\hline $\begin{array}{l}\text { 30-day mortality } \\
90 \text {-day mortality }\end{array}$ & $5(29.4)$ & $3(9.1)$ & $2(14.3)$ & 0.170 \\
\hline
\end{tabular}

* show significant associations $(p<0.05)$ 
circuits suffered COVID-19 pneumonia after discharge, and half of these died (Fig. 1). Two had been sent home and were readmitted for pneumonia 10 and 12 days after discharge; one died within 30-day follow-up, the other was alive at 90 days. The third case, transferred to a geriatric rehabilitation unit, was readmitted three weeks later and was alive at 90-day follow-up. Another died at home from COVID-19 pneumonia before 30-day follow-up; this individual had probably been infected during hospitalisation or at home by a first-degree relative, a healthcare worker who was confirmed to also have SARS-CoV-2 infection. Overall 30-day mortality for the 12 COVID-19 cases confirmed by RT-PCR of nasopharyngeal swabs was thus $25 \%$. Following separation into distinct circuits no transmitted COVID-19 infections were observed neither among the patients admitted to the non-COVID-19 circuit nor in patients with negative swabs treated in the COVID-19 circuit, in spite of cohorting. Two patients with hip fracture and COVID-19 pneumonia and positive swabs died by 90 -day follow-up; one shortly after admission and before surgery, and the other over two months after surgery and discharge to a downstream nursing care facility. Thus, 30-day mortality was higher among patients treated in 2020 before cohorting ( $p=0.016$ ), as was evident with Kaplan-Meier survival analysis (Log rank, Mantel Cox $p=0.037$ ) (Fig. 2); significance was lost when comparing patients treated after cohorting with 2018-19 control (Log rank, Mantel Cox $p=0.824$ ).

\section{Discussion}

This report shows that in spite of the pandemic, hip fractures without infection by SARS-CoV-2 can be treated safely in a designated "safe" circuit, and patients with concomitant COVID-19 pneumonia can be treated surgically if clinically stable; the characteristics and survival did not differ significantly compared with the corresponding months of previous years.

We were unable to observe increased mortality among patients with COVID-19 infection and hip fracture. Many have described high mortality with approximately one-third of patients dying in the first month after the fracture (Supplementary Table 1) [6]. However, several included preoperatively as well as postoperatively diagnosed infections, and the proportion of total infections diagnosed on admission varied between 7.7 and $70 \%$ [3, 6-11, 24]. We report the results of patients diagnosed preoperatively and treated in a separate circuit; among those treated before the creation of separate pathways, four patients (23.5\%) suffered coronavirus infectious disease-19 in the following 30 days and half of them died. Overall mortality for RT-PCR-confirmed cases was $25 \%$, quite similar to that observed by others.

Controversy remains regarding the management of patients suffering a hip fracture with coronavirus infectious disease-19 in the acute preoperative phase, especially concerning the timing of surgery and perioperative measures. Narang [25] had a lower surgical delay for COVID-19(+)
Fig. 2 Kaplan-Meier survival curves for the four groups described: historic controls treated in 2018-19 (black), pre-cohorting in 2020 (blue, dotted), non-COVID circuit of 2020 (green, dashed) and COVID-19 circuit of 2020 (red, solid). Mortality was higher among patients treated before cohorting [Log-Rank (MantelCox) $p=0.037$ ], but not after establishing separate circuits [Log-Rank (Mantel-Cox) $p=0.824]$

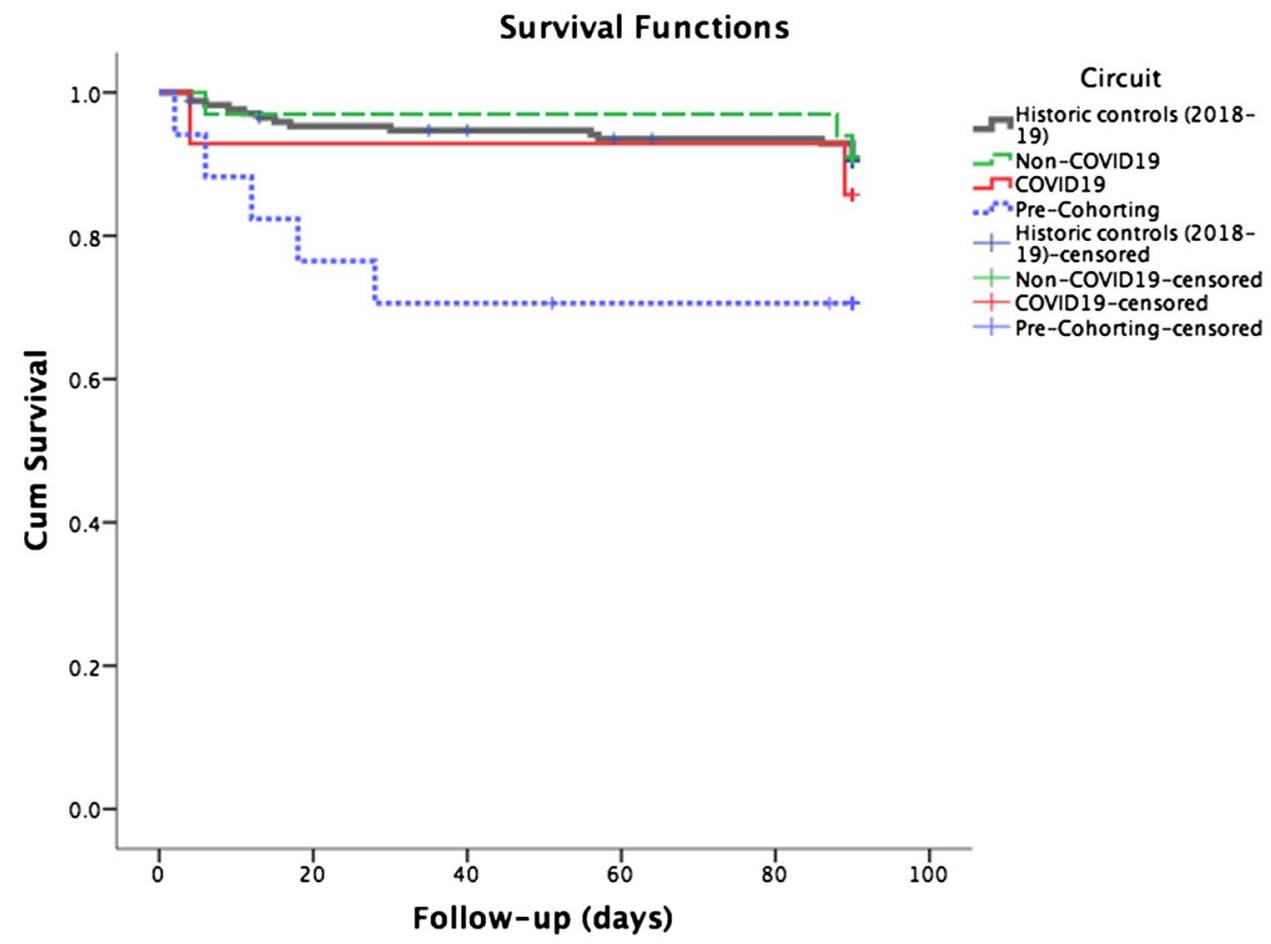


patients compared to those without infection (59.2\% in $<36$ h vs. $77.9 \%$, respectively); Egol [26] reported a longer surgical delay among cases confirmed or suspected to have COVID-19, as in our series. Other authors had surgical delays comparable to or lower than previous years [7, 27]. Many of our cases presented with viral pneumonia, fever and hypoxia; guidelines consider chest infections with systemic involvement an acceptable reason for surgical delay [28], and we considered it prudent to avoid the additional inflammatory insult of surgery on patients with systemic involvement. Long-acting regional blocks, repeated if necessary, allow patient mobilisation and facilitate care in addition to reducing opiate demand and avoiding delirium $[29,30]$. Daily discussion of cases among designated members of all specialties involved (internal medicine or geriatrics, orthopaedic surgeons, anaesthesiologists) is vital to determine the optimum moment of surgery.

Systematic reviews report similar 30-mortality and complication rates with neuroaxial and general anaesthesia for management of hip fractures [31, 32]. Depth of intraoperative sedation and avoidance of hypotension seems to be more relevant [33]. The advantages of spinal anaesthesia in patients with coronavirus infectious disease-19 are multiple, as mechanical ventilation is not necessary, aerosol-producing procedures are avoided, and the risk of contagion for the anaesthestic team is reduced. All patients treated in our COVID-19 pathway were treated with spinal anaesthesia.

The inflammatory changes in coronavirus infectious disease-19 include a prothrombotic state with high rates of venous thromboembolism (VTE) [34]. European guidelines recommend LMWH, UFH or Fondaparinux [35]. All patients included in this report received enoxaparin. All patients in the study by Egol [26] received chemical prophylaxis for VTE without specifying which, and all those reported in another single-center series from New York received LMWH and spinal anaesthesia and survived to discharge [36].

Other details regarding perioperative management are the avoidance of intraoperative tranexamic acid in COVID-19 cases, as also recommended by Egol [26], and the emphasis on early mobilisation and physiotherapy. Patients placed under contact or respiratory precautions are visited less by health care workers and suffer higher rates of anxiety, depression, and delirium [37, 38]. Mobilisation on the first postoperative day is a standard of care included in several guidelines, and a larger proportion of patients treated in the COVID-19 pathway was mobilised the day of or the day after surgery in spite of respiratory precautions.

Our study is relevant because it shows hip fracture patients can be treated safely in a dedicated circuit in spite of increased demands on the healthcare system due to the COVID-19 pandemic. The region in which this study was performed was among the ones with the highest incidence and case fatality ratios worldwide at the time of data collection, with COVID-19-related hospitalisations occupying over $100 \%$ of acute care beds available. In spite of this caseload, we observed no secondary COVID-19 infections once the separate "clean" circuit was established. All patients admitted to hospital, regardless of diagnosis, were screened for COVID-19 form March 14th on; other authors only tested clinically suspicious cases and it is likely that they may have not detected several asymptomatic cases, as was the case for over one-third of our RT-PCR $(+)$ patients. A study from New York reported a $12 \%$ positive rate for SARS-CoV-2 once the universal screening was adopted during the first pandemic wave, with over half of the detected cases asymptomatic at the time of admission [39], and 11\% of the patients that tested positive at admission in a multicentric series from London were asymptomatic [40]. Consequently, other authors may have a selection bias that could overestimate mortality.

Our study has several limitations. It is limited to a single centre, and while the case number of COVID-19 positive or suspected patients was low, it was similar or higher than many other studies. We cannot rule out that our study is underpowered to elucidate factors associated with poor outcomes such as increased mortality, and multicentre studies are more likely to detect relevant associations. Adjusted statistical analysis was not possible for the same reasons. We believe that our results are relevant, especially regarding the fact that mortality was similar among the patients treated in the COVID-19 and nonCOVID-19 pathways, compared to the 2018-19 controls (7.1\% and $3.0 \%$ vs. $5.2 \% 30$-day mortality, respectively), particularly for future systematic reviews and meta-analyses. Second, different clinicians from the same hospital treated the COVID-19 and Non-COVID-19 circuits, a potential source of intervention bias. Each case was addressed individually by physicians experienced in multidisciplinary fracture care, and guidelines and recommendations for management of coronavirus infectious disease-19 changed quickly during the observation period. The stress suffered by the local healthcare system conditioned the availability of operating theatres and critical care beds as well as the availability of downstream rehabilitative and geriatric care. In spite of these limitations, average surgical delay was lower in 2020 compared to the previous years, as was also the length of stay. Lower length of stay in the pre-cohorting period, as well as among non-COVID19 patients, was driven largely by two reasons: on the one hand, beds needed to be urgently liberated to accommodate the increasing number of COVID19 patients. On the other hand, patients were discharged home earlier in an effort to avoid nosocomial transmission. In spite of shortening length of stay, nosocomial transmission could not be completely avoided, and nearly one in four patients suffered coronavirus infectious disease-19, accounting for two of the five deaths observed at 30-day follow-up. The other three patients died in-hospital, which could possibly be attributable 
to the breakup of co-managed orthogeriatric care in the early stages of the pandemic, due to insufficient staffing-all geriatricians fell ill during this period. Third, we only collected follow-up data regarding 30-day and 90-day mortality and secondary COVID-19 diagnosis at 30 days. Other outcome measures such as return to activities of daily living or baseline housing, as well as PROMs, are relevant for patients; it is likely that the lack of downstream rehabilitative and geriatric care in 2020 negatively affected mid-term recovery.

In conclusion, we were unable to detect increased mortality rates among patients admitted for hip fractures with preoperatively confirmed or suspected coronavirus infectious disease-19 as compared to COVID-19-negative cases observed in the same time period, and also as compared to the corresponding periods in 2018-19. Dedicated and separate circuits for patients with and without coronavirus infectious disease-19 provided adequate hip fracture care in spite of the stress suffered by the healthcare system during the first wave of the COVID-19 pandemic. Delaying surgery among patients with severe respiratory illness until a favourable trend could be observed did not lead to increased mortality.

Supplementary Information The online version contains supplementary material available at https://doi.org/10.1007/s41999-021-00455-x.

Funding There is no funding source.

Availability of data and materials Upon request.

\section{Compliance with ethical standards}

Conflict of interest The authors declare that they have no conflict of interest.

Ethical approval We obtained institutional review board approval for this study, defined as non-intervention by the Spanish Medicines Agency (Agencia Española de Medicamentos y Productos Sanitarios, AEMPS).

Informed consent Informed consent for participation was not considered necessary for this type of audit, as per local legislation.

\section{References}

1. Mathai NJ, Venkatesan AS, Key T et al (2020) COVID-19 and orthopaedic surgery: evolving strategies and early experience. Bone Joint Open 1:160-166. https://doi.org/10.1302/20463758.15.BJO-2020-0021.R1

2. Luengo-Alonso G, Pérez-Tabernero FG-S, Tovar-Bazaga M et al (2020) Critical adjustments in a department of orthopaedics through the COVID-19 pandemic. Int Orthop. https://doi. org/10.1007/s00264-020-04647-1

3. Nuñez JH, Sallent A, Lakhani K et al (2020) Impact of the COVID-19 pandemic on an emergency traumatology service: experience at a tertiary trauma centre in Spain. Injury 51:14141418. https://doi.org/10.1016/j.injury.2020.05.016
4. Scott CEH, Holland G, Powell-Bowns MFR et al (2020) Population mobility and adult orthopaedic trauma services during the COVID-19 pandemic: fragility fracture provision remains a priority. Bone Joint Open 1:182-189. https://doi. org/10.1302/2046-3758.16.BJO-2020-0043.R1

5. Lim MA, Pranata R (2020) Coronavirus disease 2019 (COVID19) markedly increased mortality in patients with hip fracturea systematic review and meta-analysis. J Clin Orthop Trauma. https://doi.org/10.1016/j.jcot.2020.09.015

6. Hall AJ, Clement ND, Farrow L et al (2020) IMPACT-Scot report on COVID-19 and hip fractures. Bone Joint J 102B:1219-1228. https://doi.org/10.1302/0301-620X.102B9.BJJ2020-1100.R1

7. Dupley L, Oputa TJ, Bourne JT, North West COVID NOF Study Group (2020) 30-day mortality for fractured neck of femur patients with concurrent COVID-19 infection. Euro J Orthop Surg Traumatol Orthop Traumatol. https://doi.org/10.1007/s0059 0-020-02778-0

8. LeBrun DG, Konnaris MA, Ghahramani GC et al (2020) Hip fracture outcomes during the COVID-19 pandemic: early results from New York. J Orthop Trauma 34:403-410. https://doi.org/10.1097/ BOT.0000000000001849

9. Cheung ZB, Forsh DA (2020) Early outcomes after hip fracture surgery in COVID-19 patients in New York City. J Orthop 21:291-296. https://doi.org/10.1016/j.jor.2020.06.003

10. Archer JE, Kapoor S, Piper D, Odeh A (2020) The impact of COVID-19 on 30-day mortality in patients with neck of femur fractures. Bone Joint Open 1:326-329

11. Muñoz Vives JM, Jornet-Gibert M, Cámara-Cabrera J et al (2020) Mortality rates of patients with proximal femoral fracture in a worldwide pandemic: preliminary results of the Spanish HIPCOVID observational study. J Bone Joint Surg Am 102:e69. https ://doi.org/10.2106/JBJS.20.00686

12. Cacciapaglia G, Cot C, Sannino F (2020) Second wave COVID19 pandemics in Europe: a temporal playbook. Scientific Reports 10:15514. https://doi.org/10.1038/s41598-020-72611-5

13. Currie C (2018) Hip fracture audit: creating a "critical mass of expertise and enthusiasm for hip fracture care"? Injury 49:1418 1423. https://doi.org/10.1016/j.injury.2018.06.025

14. Bello X, Pardo-Seco J, del Molino-Bernal MLP et al (2020) Phylogeography of SARS-CoV-2 pandemic in Spain: a story of multiple introductions, micro-geographic stratification, founder effects, and super-spreaders. Zool Res 41:1-16

15. España De, Juan Carlos I, Rey. (1986) Ley 14/1986, de 25 de abril, General de Sanidad. Boletín Oficial del Estado, Spain

16. Condes E, Arribas JR (2020) Impact of COVID-19 on Madrid hospital system. Enfermedades Infecciosas y Microbiología Clínica. https://doi.org/10.1016/j.eimc.2020.06.005

17. Katz S (1983) Assessing self-maintenance: activities of daily living, mobility, and instrumental activities of daily living. J Am Geriatr Soc 31:721-727. https://doi.org/10.1111/j.1532-5415.1983. tb03391x

18. Pfeiffer E (1975) A short portable mental status questionnaire for the assessment of organic brain deficit in elderly patients. J Am Geriatr Soc 23:433-441

19. Bjorgul K, Novicoff WM, Saleh KJ (2010) American Society of Anesthesiologist Physical Status score may be used as a comorbidity index in hip fracture surgery. J Arthroplasty 25:134-137. https://doi.org/10.1016/j.arth.2010.04.010

20. Pollán M, Pérez-Gómez B, Pastor-Barriuso R et al (2020) Prevalence of SARS-CoV-2 in Spain (ENE-COVID): a nationwide, population-based seroepidemiological study. Lancet 396:535-544. https://doi.org/10.1016/S0140-6736(20)31483-5

21. Medicine AS of RA and P Practice Recommendations on Neuraxial Anesthesia and Peripheral Nerve Blocks during the COVID-19 Pandemic. https://www.asra.com/page/2905/practice-recommenda 
tions-on-neuraxial-anesthesia-and-peripheral-nerve-blocks-dur. Accessed 6 Oct 2020

22. Aranda-Narváez J, Tallón-Aguilar L, Pareja-Ciuró F, et al (2020) Emergency Surgery and Trauma Care During COVID-19 Pandemic. Recommendations of the Spanish Association of Surgeons. Cirugía Española (English Edition) https://doi.org/10.1016/j.ciren g.2020.09.015

23. Caeiro-Rey JR, Ojeda-Thies C, Cassinello-Ogea C et al (2020) COVID-19 y fractura por fragilidad de la cadera. Recomendaciones conjuntas de la Sociedad Española de Fracturas Osteoporóticas y la Sociedad Española de Geriatría y Gerontología. Rev Esp Geriatr Gerontol 55:300-308. https://doi.org/10.1016/j. regg.2020.07.001

24. Macey ARM, Butler J, Martin SC et al (2020) 30-day outcomes in hip fracture patients during the COVID-19 pandemic compared to the preceding year. Bone Joint Open 1:415-419. https://doi. org/10.1302/2046-3758.17.BJO-2020-0077.R1

25. Narang A, Chan G, Aframian A et al (2020) Thirty-day mortality following surgical management of hip fractures during the COVID-19 pandemic: findings from a prospective multi-centre UK study. Int Orthop. https://doi.org/10.1007/s00264-020-04739 $-\mathrm{y}$

26. Egol KA, Konda SR, Bird ML et al (2020) Increased mortality and major complications in hip fracture care during the COVID19 pandemic: a New York City perspective. J Orthop Trauma 34:395-402. https://doi.org/10.1097/BOT.0000000000001845

27. Greenhalgh M, Dupley L, Unsworth R, Boden R (2020) Where did all the trauma go? A rapid review of the demands on orthopaedic services at a UK Major Trauma Centre during the COVID-19 pandemic. Int J Clin Pract. https://doi.org/10.1111/ijcp.13690

28. Griffiths R, Alper J, Beckingsale A et al (2012) Management of proximal femoral fractures 2011. Anaesthesia 67:85-98

29. Ritcey B, Pageau P, Woo MY, Perry JJ (2016) Regional nerve blocks for hip and femoral neck fractures in the emergency department: a systematic review. CJEM 18:37-47. https://doi. org/10.1017/cem.2015.75

30. Garlich JM, Pujari A, Debbi EM et al (2020) Time to block: early regional anesthesia improves pain control in geriatric hip fractures. J Bone Joint Surg Am 102:866-872. https://doi. org/10.2106/JBJS.19.01148

31. O'Donnell CM, McLoughlin L, Patterson CC et al (2018) Perioperative outcomes in the context of mode of anaesthesia for patients undergoing hip fracture surgery: systematic review and meta-analysis. Br J Anaesth 120:37-50. https://doi.org/10.1016/j. bja.2017.09.002

32. White SM, Moppett IK, Griffiths R et al (2016) Secondary analysis of outcomes after 11,085 hip fracture operations from the prospective UK Anaesthesia Sprint Audit of Practice (ASAP-2). Anaesthesia 71:506-514. https://doi.org/10.1111/anae.13415

33. Sieber FE, Neufeld KJ, Gottschalk A et al (2018) Effect of depth of sedation in older patients undergoing hip fracture repair on postoperative delirium: the STRIDE Randomized Clinical Trial. JAMA Surg 153:987-995. https://doi.org/10.1001/jamas urg.2018.2602

34. Dobesh PP, Trujillo TC (2020) Coagulopathy, venous thromboembolism, and anticoagulation in patients with COVID-19. Pharmacother J Hum Pharmacol Drug Ther. https://doi.org/10.1002/ phar.2465

35. Flevas DA, Megaloikonomos PD, Dimopoulos L et al (2018) Thromboembolism prophylaxis in orthopaedics: an update. EFORT Open Rev 3:136-148. https://doi.org/10.1302/20585241.3.170018

36. Muse IO, Montilla E, Gruson KI, Berger J (2020) Perioperative management of patients with hip fractures and COVID-19: A single institution's early experiences. J Clin Anesth 67:110017. https ://doi.org/10.1016/j.jclinane.2020.110017

37. Sprague E, Reynolds S, Brindley P (2016) Patient isolation precautions: are they worth it? Can Respir J. https://doi. org/10.1155/2016/5352625

38. Kirkland KB, Weinstein JM (1999) Adverse effects of contact isolation. Lancet 354:1177-1178. https://doi.org/10.1016/S0140 -6736(99)04196-3

39. Gruskay JA, Dvorzhinskiy A, Konnaris MA et al (2020) Universal testing for COVID-19 in essential orthopaedic surgery reveals a high percentage of asymptomatic infections. J Bone Joint Surg 102:1379-1388. https://doi.org/10.2106/JBJS.20.01053

40. Kayani B, Onochie E, Patil V et al (2020) The effects of COVID-19 on perioperative morbidity and mortality in patients with hip fractures. Bone Joint J 102:1136-1145. https://doi. org/10.1302/0301-620X.102B9.BJJ-2020-1127.R1

Publisher's Note Springer Nature remains neutral with regard to jurisdictional claims in published maps and institutional affiliations. 